\title{
Non-inverted pedicle internal limiting membrane transposition for large macular holes
}

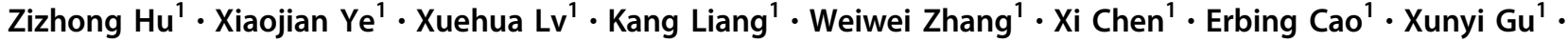 \\ Qinghuai Liu $\mathbb{B}^{1} \cdot$ Ping Xie $^{1}$
}

Received: 23 November 2017 / Revised: 7 April 2018 / Accepted: 12 April 2018 / Published online: 29 May 2018

(c) The Royal College of Ophthalmologists 2018

\begin{abstract}
Purpose The purpose of this study is to investigate the effectiveness of a new surgical technique of non-inverted pedicle internal limiting membrane (ILM) transposition for the treatment of eyes with large macular hole.

Methods This is a retrospective, consecutive, interventional case series. Twelve eyes of 12 consecutive patients who underwent vitrectomy for the treatment of a large macular hole (MH size $>400 \mu \mathrm{m})$ were treated. ILM was peeled and left with a pedicle attached to the superior temporal retina. The macular hole was covered by transposition of the pedicle ILM in a non-inverted way. Preoperative and postoperative best-corrected visual acuity (BCVA), SD-OCT image, macular sensitivity by microperimetry, and multifocal electroretinogram (mERG) response were evaluated. All of the patients were followed for more than 3 months.

Results Postoperative OCT examination confirmed 11 of 12 macular hole closed (91.7\%). Six macular hole filled with silicone oil closed as early as the next day. The postoperative BCVA significantly increased compared with preoperative BCVA $(P=0.002)$. The improvement of macular sensitivity within $2^{\circ}$ and $8^{\circ}$ circle was also statistical significant $\left(P=0.018\right.$ and $P=0.017$, respectively). Fixation stability, shown as the percentage of fixation point within the $2^{\circ}$ circle and $4^{\circ}$ circle, was remarkably improved $(P=0.017$ and $P=0.018$, respectively). The $\mathrm{R} 1 / \mathrm{R} 2$ and $\mathrm{R} 1 / \mathrm{R} 4$ ring ratios also increased significantly as compared with that of baseline.

Conclusion These findings indicate that the non-inverted pedicle ILM transposition results in a high incidence of anatomic closure with good visual outcome for the treatment of large macular hole.
\end{abstract}

\section{Introduction}

Although vitrectomy combined with internal limiting membrane (ILM) removal is considered a standard procedure for idiopathic macular hole (IMH), the surgical closure is challenging in cases of long-lasting, recurrent, or large macular holes (MHs) [1]. Recently, different surgical techniques have been introduced to improve the closure

Electronic supplementary material The online version of this article (https://doi.org/10.1038/s41433-018-0107-2) contains supplementary material, which is available to authorized users.

Qinghuai Liu

liuqh@njmu.edu.cn

$\triangle$ Ping Xie

xieping9@126.com

1 Department of Ophthalmology, The First Affiliated Hospital of Nanjing Medical University, Nanjing, China rates of such cases with variable success [2, 3]. The inverted ILM flap technique, first reported by Michalewska et al. [4], has shown improved functional and anatomical outcomes for those cases. This technique was then modified by injecting viscoelastic into the $\mathrm{MH}$ as a "viscoelastic cap" or employing autologous gluconated blood clumps as a "macular plug" to facilitate the closure of MH [5, 6]. For the recurrent MH, Park et al. [7] used a free ILM flap, which was collected from the residual ILM, to cover the MH with the assistance of perfluoro- $n$-octane. However, either side of the free ILM flap could not be easily identified during surgery.

Here we described and evaluated the effectiveness of a new technique that created a non-inverted pedicle ILM and transposes it to cover the $\mathrm{MH}$.

\section{Patients and methods}

This was a retrospective, consecutive, interventional study conducted in the First Affiliated Hospital of Nanjing 
A

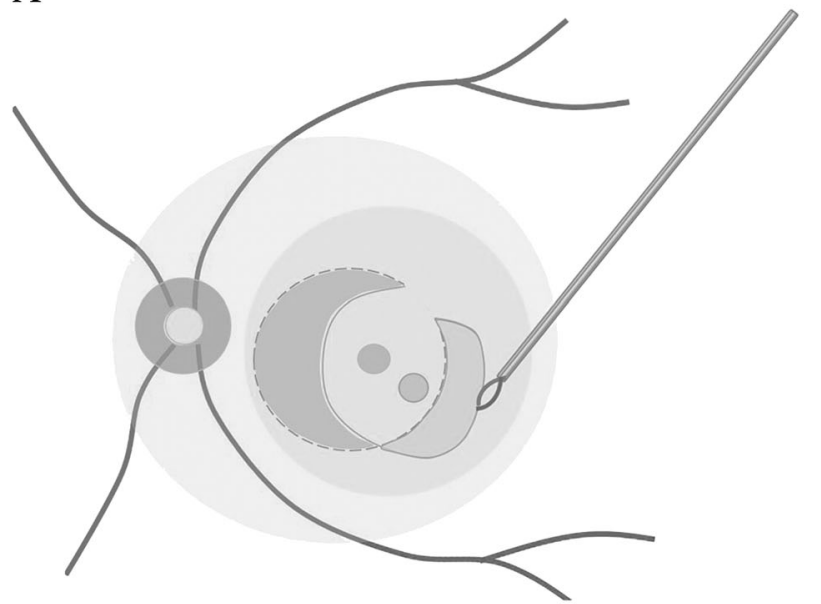

B

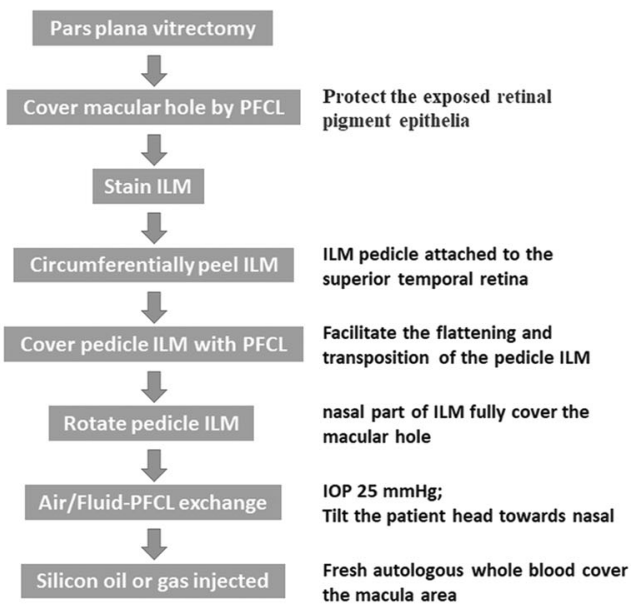

introduced into the eye to facilitate the transposition and flattening of pedicle ILM (green). The free temporal edge of the ILM was grasped and the whole pedicle ILM was rotated till the nasal part of ILM fully cover the macular hole

was introduced into the eye with an amount sufficient to cover the pedicle ILM as a single large bubble above the posterior pole, in purpose of facilitating the flattening and transposition of the pedicle ILM. The free temporal edge of the ILM was grasped and the whole pedicle ILM was rotated till the nasal part of ILM fully cover the $\mathrm{MH}$ (Fig. 1). With the assistance of PFCL, the curly edge of ILM was flattened gently by forceps. In the end, air/FluidPFCL exchange was carefully performed and the PFCL was aspirated as completely as possible.

Initially, silicon oil (Oxane 5700, Bausch \& Lomb) tamponade were performed in eight cases to avoid the displacement of the transposition ILM, which also facilitated the early visualization of macular morphological change. In the following three cases, autologous whole blood was applied and 15\% perfluoropropane (C3F8) was injected to fill the eye. The autologous whole blood was obtained from the patient's antecubital vein and immediately injected to cover the macula. The fresh autologous whole blood soon became a clot to cover the macula area. After surgery, patients were requested to remain in a prone position for 3 day and avoid the supine position. Closure of MH was assessed using spectral domain optical coherence tomography (OCT). In those cases tamponaded with silicone oil, silicone oil was removed 1 month after the closure of the MH.

\section{Examinations}

The presence of a large IMH was confirmed by spectraldomain (SD)-OCT (Cirrus HD-OCT; Carl Zeiss Meditec, Inc., Dublin, CA). The eyes with myopic $\mathrm{MH}$ or 
Table 1 Characteristics of patients with large MH

\begin{tabular}{|c|c|c|c|c|c|c|c|c|c|c|}
\hline \multirow{2}{*}{$\begin{array}{l}\text { Case } \\
\text { no. }\end{array}$} & \multirow[t]{2}{*}{ Age } & \multirow[t]{2}{*}{ Gender } & \multirow{2}{*}{$\begin{array}{l}\text { MH aperture } \\
\text { diameter }(\mu \mathrm{m})\end{array}$} & \multirow{2}{*}{$\begin{array}{l}\text { MH base diameter } \\
(\mu \mathrm{m})\end{array}$} & \multirow[t]{2}{*}{ Dye } & \multirow{2}{*}{$\begin{array}{l}\text { Intraocular } \\
\text { temponade }\end{array}$} & \multirow{2}{*}{$\begin{array}{l}\text { Follow- } \\
\text { up } \\
\text { (months) }\end{array}$} & \multirow{2}{*}{$\begin{array}{l}\text { Hole closure } \\
\text { (days) }\end{array}$} & \multicolumn{2}{|c|}{ BCVA (logMAR) } \\
\hline & & & & & & & & & Pre & Post \\
\hline 1 & 46 & $\mathrm{~F}$ & 448 & 1026 & ICG & Silicon oil & 8 & 1 & 2 & 0.6 \\
\hline 2 & 64 & $\mathrm{~F}$ & 914 & 1108 & ICG & Silicon oil & 8 & 1 & 1 & 0.52 \\
\hline 3 & 64 & $\mathrm{~F}$ & 746 & 1509 & ICG & Silicon oil & 8 & No & 1 & 1 \\
\hline 4 & 77 & $\mathrm{~F}$ & 695 & 972 & ICG & Silicon oil & 7 & 1 & 1 & 0.8 \\
\hline 5 & 75 & M & 656 & 1073 & BBG & Silicon oil & 7 & 1 & 1 & 0.4 \\
\hline 6 & 64 & F & 408 & 744 & BBG & Silicon oil & 7 & 1 & 0.6 & 0.16 \\
\hline 7 & 33 & M & 729 & 1514 & BBG & Silicon oil & 8 & 7 & 2.3 & 2.0 \\
\hline 8 & 66 & M & 441 & 817 & BBG & Silicon oil & 8 & 1 & 0.92 & 0.7 \\
\hline 9 & 58 & M & 821 & 1115 & BBG & Silicon oil & 8 & 30 & 1.3 & 0.7 \\
\hline 10 & 70 & $\mathrm{~F}$ & 672 & 1194 & BBG & $\mathrm{C} 3 \mathrm{~F} 8$ & 5 & 14 & 1.3 & 0.7 \\
\hline 11 & 77 & $\mathrm{~F}$ & 548 & 1033 & BBG & C3F8 & 4 & 14 & 0.82 & 0.7 \\
\hline 12 & 63 & F & 816 & 1320 & BBG & C3F8 & 5 & 14 & 1 & 0.7 \\
\hline Overall & $62.1 \pm 13.7$ & $F(8 / 12)$ & $657.8 \pm 164.4$ & $1118.8 \pm 238.5$ & ICG (4/12) & C3F8 (3/12) & $6.9 \pm 1.4$ & $11 / 12(91.7 \%)$ & $1.23 \pm 0.50$ & $0.67 \pm 0.48$ \\
\hline
\end{tabular}

$B B G$ Blilliant Blue G, BCVA best-corrected visual acuity, $I C G$ indocyanine green, logMAR logarithm of the minimal angle of resolution, $M H$ macular hole

preoperative $\mathrm{MH}$ retinal detachment, or choroidal neovascularization, or macular atrophy were excluded. Furthermore excluded were those with a history of other retinal disorders such as such as severe cataract, diabetic retinopathy, or retinal vein occlusion that could affect the central vision. All of the patients had postoperative follow-up periods of at least 3 months.

All patients had a complete ophthalmic evaluation before and after surgery, including SD-OCT images, best-corrected visual acuity (BCVA), microperimetry and multifocal electroretinogram (mERG). We recorded Snellen charts to measure BCVA and BCVA was converted to logarithm of minimum angle of resolution (logMAR) for statistical calculation. The retinal sensitivity was determined with the microperimetry MP1 (software version, 1.4.2.SP1). The Goldmann III, 4-2 staircase strategy was adopted and the sensitivity of 24 locations within the central $8^{\circ}$ was evaluated. Roland-RETI scan system (Roland Consult, Brandenburg, Germany) was employed to record the multifocal ERG was carried out under the guidelines of the International Society for Clinical Electrophysiology for Vision (ISCEV) 2007 edition [8]. The stimulation source was a CRT monitor with a $75 \mathrm{~Hz}$ frame rate. Stimulation calibrations were performed as provided by the RETI-scan software (Roland Consult). The cutoff values were $5 \mathrm{~Hz}$ and $100 \mathrm{~Hz}$ for the high and low pass, respectively. The artifact level was $10 \%$.

\section{Statistical analysis}

SPSS software version 20.0 (SPSS, Inc., Chicago, IL) was used to perform statistical analyses of the data. The nonparametric paired Wilcoxon signed-rank test was used to compare values between before and after surgery. $P$-value $<0.05$ was considered statistically significant.

\section{Results}

To date, we have performed the non-inverted pedicle ILM transposition technique on 12 consecutive eyes. The preoperative baseline characteristics of all cases were summarized in Table 1. The mean aperture diameter of MHs was $657.8 \pm 164.4 \mu \mathrm{m}$ (range, $408-914 \mu \mathrm{m}$ ). The mean base diameter of MHs was $1118.8 \pm 238.5 \mu \mathrm{m}$ (range, 744-1514 $\mu \mathrm{m})$.

No intraoperative or postoperative complications related to the pedicle ILM transposition technique were noted. After a mean follow-up duration of 6.9 months (range, 4-8 months), 11 of the 12 eyes yielded with a "V" shape MH closure (closure rate $91.7 \%$ ). For eyes filled with silicon oil, closure of MH was observed as early as the next day in six eyes postoperatively (Fig. 2, Patient 1, 2, 4, 5, 6, 8). Another 2 patients showed initial MH reduction in size 1 day post operation and MH closure 5 days (Fig. 2, Patient 7) and 30 days post operation (Fig. 2, Patient 9). In one eye, the intraoperative ILM transposition was not completed and the MH showed a "W" closure, in which foveal defect of the neurosensory retina with RPE surface exposure (Fig. 2). The transposition ILM was confirmed covering the MH by BBG stain in the end of the removal of the silicon oil (Fig. 2). Regarding three eyes filled with C3F8, OCT images were not available until 2 weeks, when the gas were mostly absorbed and MH closure were confirmed.

The pre- and postoperative visual quality were assessed by BCVA, microperimetry, and multifocal ERG (Fig. 3). As 

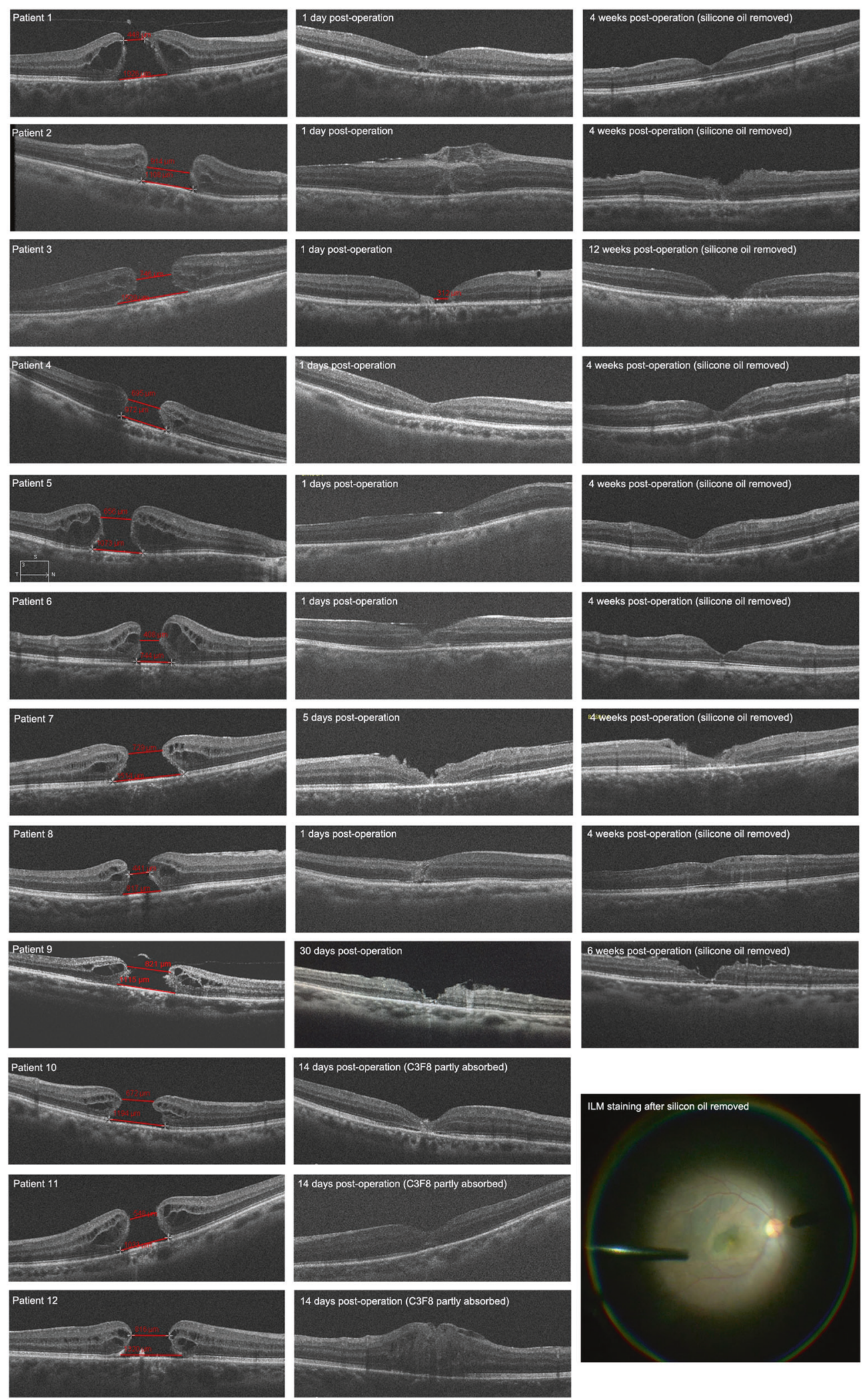

shown in Table 2, the BCVA converted as LogMAR was $1.23 \pm 0.50$ at baseline and improved to $0.67 \pm 0.48$ at the last follow-up after surgery $(P=0.002)$. Macular sensitivity, assessed by MP1, increased from $1.45 \pm 2.94$ to $6.33 \pm 4.90 \mathrm{~dB}$ within the $2^{\circ}$ circle $(p=0.008)$, and $3.14 \pm$ 4.51 to $8.91 \pm 5.52 \mathrm{~dB}$ within the $8^{\circ}$ circle $(p=0.008)$ 
Fig. 2 Changes in optical coherence tomographic (OCT) findings after vitrectomy with non-inverted pedicle internal limiting membrane (ILM) transposition for large macular hole. Preoperative OCT indicates the aperture and basal diameters of the large macular holes. From patients 1 to 9 , eyes were filled with silicone oil after surgery. Closure of macular hole was observed as early as the next day in 6 eyes postoperatively (Patient 1, 2, 4, 5, 6, and 8). Another 2 patients showed initial macular hole reduction in size 1 day post operation and macular hole closure 5 days (Patient 7), and 30 days post operation (Patient 9). In one eye, the intraoperative ILM transposition was not completed and the macular hoel showed a "W" closure, in which foveal defect of the neurosensory retina with RPE surface exposure (Patient 3 ). Three eyes filled with $\mathrm{C} 3 \mathrm{~F} 8$, OCT images were not available until 2 weeks, when the gas were mostly absorbed and macular hole closure were confirmed (Patient 10, 11, and 12). In the secondary surgery of silicon oil removal, the transposition ILM was confirmed covering the macular hole by Brilliant Blue-G stain (Arrow, Patient 8)

(Table 2). As for fixation stability, a significant improvement of the percentage of fixation points within the $2^{\circ}$ circle (from $54.6 \pm 33.9 \%$ to $94.4 \pm 7.85 \% ; p=0.017$ ) and $4^{\circ}$ circle (from $78.3 \pm 30.6 \%$ to $99.6 \pm 0.51 \%$; $p=0.018$ ) was assessed compared baseline and last time point. Regarding mERG analysis, although we found no statistically significant change of the $\mathrm{mERG}$ response density after surgery in the central (R1), para-central (R2), R3, R4, and R5, the $\mathrm{R} 1 / \mathrm{R} 2$ and $\mathrm{R} 1 / \mathrm{R} 4$ ring ratios were increased significantly at the last time point as compared with that of baseline (R1/ $\mathrm{R} 2$ : from $1.39 \pm 0.60$ to $3.23 \pm 1.35, p=0.028$; $\mathrm{R} 1 / \mathrm{R} 4$ : from $3.66 \pm 1.75$ to $6.56 \pm 2.30, p=0.043)$.

\section{Discussion}

The prominent feature of this technique is to release the macular traction by ILM peeling and cover the $\mathrm{MH}$ by transposition of a non-inverted pedicle ILM. ILM peeling has been demonstrated with higher $\mathrm{MH}$ closure rates and functional recovery because of the elimination of its tractional forces at the vitreoretinal interface [9]. In our technique, after peeling, the ILM was not removed off but left with a pedicle to cover the $\mathrm{MH}$. We considered a singlelayered and non-inverted ILM would be a more physiological and regular structure for glia proliferation, macular closure, and regeneration of inner and outer retina.

For those large, chronic, or recurrent macualr holes, peeling the ILM seems not enough for the successful $\mathrm{MH}$ closure. Based on inverted ILM technique by Michalewska et al. [4], modified techniques have been subsequently developed, which all yielded high $\mathrm{MH}$ closure rate [5, 10]. The inverted ILM flap, as a key step, is considered to be capable of stimulating the proliferation of glial cells to fill MHs. Later, Michalewska et al. [11] and Park SW et al. [12] proposed using a true single-layered ILM flap to cover instead of filling the MH. They collected the ILM flap around or away from the MH. For ILM attached to the macular margin and flipped to cover the MH, the ILM is inverted. For ILM flap peeled away from MH, surgeons could not identify either side of the ILM during the translocation. In our technique, we peeled but not removed the ILM around the MH. Instead, the pedicle of the ILM attached to the retina allows us to cover the $\mathrm{MH}$ in a noninverted way, which would be more physiological. Gelman et al. [13] have reviewed some retinal damage induced by ILM removal including damage to müller cell, dissociated optic nerve fiber layer, and phototoxic damage. A number of studies have also investigated the histopathological characteristic of ILM [14, 15]. The ILM is characterized by a smooth vitreal side and an undulated retinal side, and the retinal side is found with müller cell debris on removed ILM specimens. Therefore, the retinal side of the ILM, if to cover the $\mathrm{MH}$, would theoretically provide a more favorable structure for glia proliferation and macular closure. Some publications have indicated decreased retinal sensitivity associated with ILM peeling [16, 17]. More recently, Tian et al. [18] have reported a novel technique for IMH with a diameter $<600 \mathrm{~mm}$, which relieved the tractive force through the ILM peeling and preserved the integrity of retina simultaneously through the ILM reposition. In our study, the BCVA, retinal sensitivity, and $\mathrm{mERG}$ response all significantly improved at the last time-point, which should attribute to the healing of the retinal layers, and might to the attachment of the peeled ILM on the macular.

In the eight successful cases, we applied silicon oil to promote the fixation of ILM on the $\mathrm{MH}$. Its transparent property enables us to visualize the early postoperative macular change. With the non-inverted ILM transposition, the $\mathrm{MH}$ started to close soon after surgery and the closure process completed < 1 day $(16 \mathrm{~h})$ in six eyes. Afterwards, we improved our technique by employing autologous blood clot to facilitate the ILM reposition. The fresh blood soon became a clot on the surface of the ILM within 1-2 min and followed by intravitreous injection of C3F8. Autologous blood clot has also been reported to assist the inverted ILM technique [6] and the treatment of $\mathrm{MH}$ retinal detachment [19]. Our findings indicated that silicon oil could be replaced by autologous blood clot to avoid the displacement of the transposition ILM after surgery. The C3F8 used in our technique avoids secondary surgery for removal of silicon oil.

In one failed case (Patient 3), the ILM transposition was not successfully completed, because the pedicle ILM was inadvertently aspirated by the 23-gauge cannula during the end of fluid /gas exchange. Later, we optimized the fluid/gas exchange by (1) reducing the intraocular pressure to around $25 \mathrm{~mm} \mathrm{Hg}$ in order to not "blow" it up and (2) tilting the patient head toward nasal cavity so as to accumulate the fluid on the nasal retina. After the $\mathrm{MH}$ closure, we removed the silicon oil and confirmed that the 
A

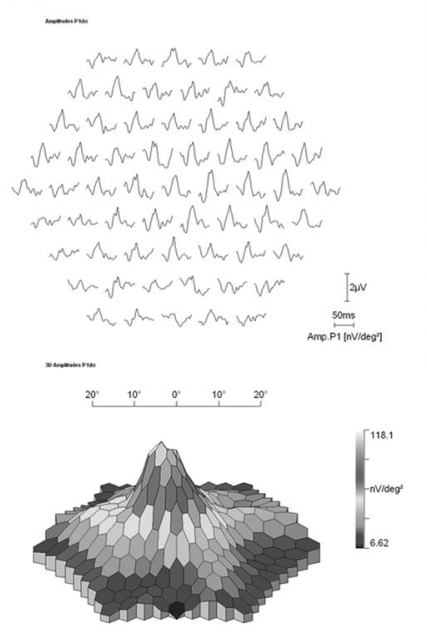

C

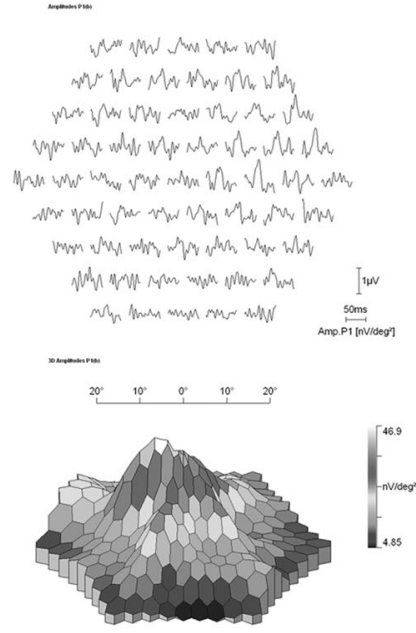

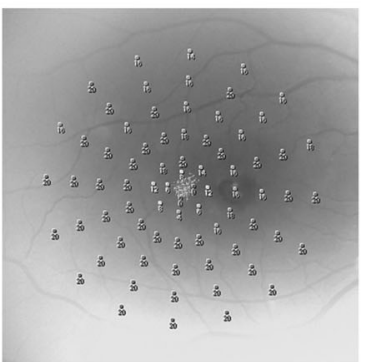

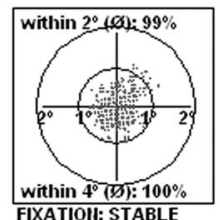

WIXATIOI: STABLF
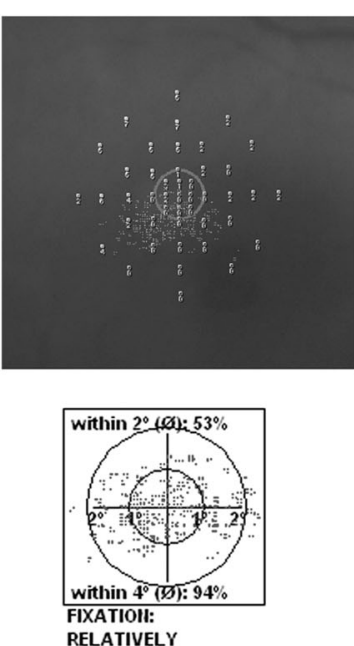

B
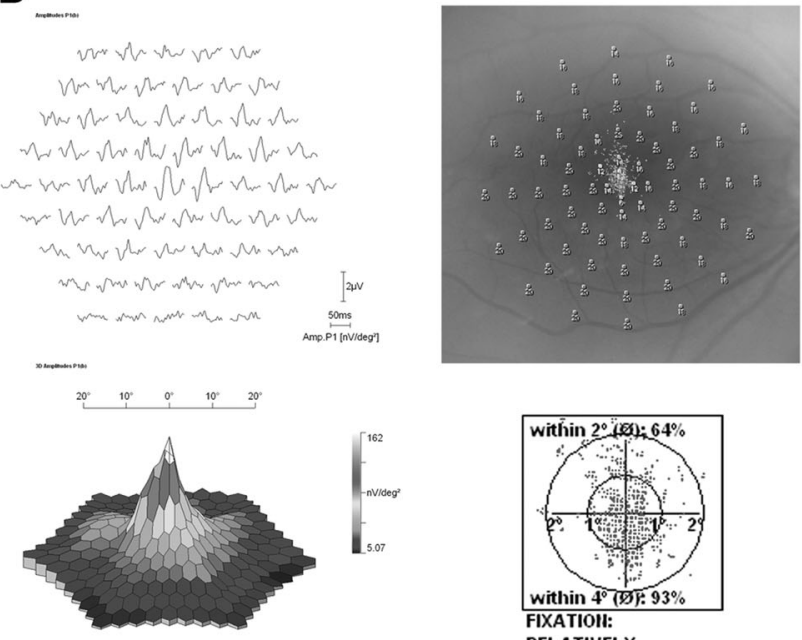

FIXATIOI:

D
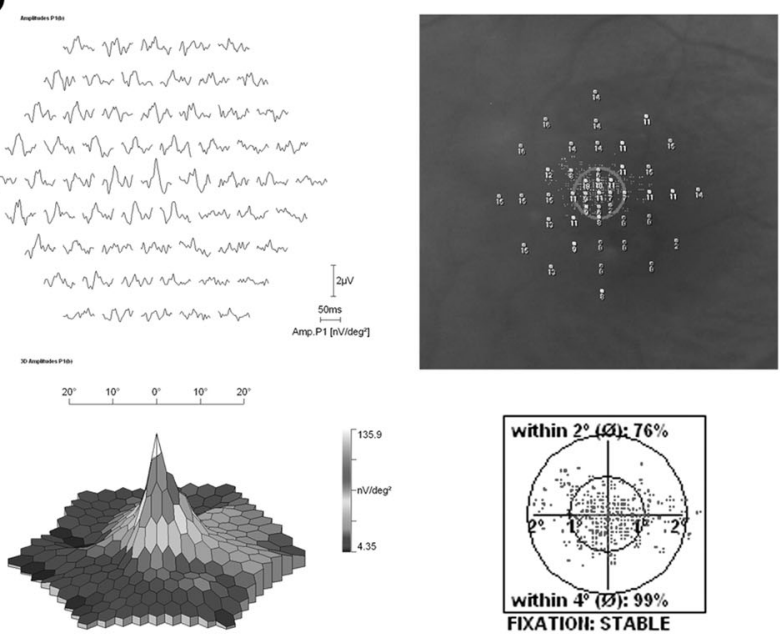

Fig. 3 Representative examples of pre- and postoperative visual quality assessed by microperimetry and multifocal electroretinogram. a Preoperative record of the right eye of a 46-year-old woman (Patient 1) with a b-wave amplitude of $118 \mathrm{nv} / \mathrm{deg}^{2}$ by multifocal ERG, shifted fixation point and some blind spots by microperimetry. The logMAR best-corrected visual acuity (BCVA) was 2.0. b At the last follow-up ( 8 months). The b-wave amplitude increased to $162 \mathrm{nv} / \mathrm{deg}^{2}$ with no shifted fixation point and blind spot. The BCVA improved to 0.6. $\mathbf{c}$ Preoperative record of the right eye of a 70-year-old woman (Patient 10) with a b-wave amplitude of $46.9 \mathrm{nv} / \mathrm{deg}^{2}$, a lot of blind spots by microperimetry, and 53\% of fixation points within the $2^{\circ}$ circle. The logMAR best-corrected visual acuity (BCVA) was 1.3. d At the last follow-up (5 months). The b-wave amplitude increased to $135.9 \mathrm{nv} / \mathrm{deg}^{2}$ with much less blind spots and improved fixation ability ( $76 \%$ within $2^{\circ}$ circle). The BCVA improved to 0.7

rotated ILM covered the macula central fovea through intraoperative BBG stain.

Nevertheless, we acknowledge that this study has several limitations. First, some surgical procedures were not consistent among those cases such as stain dye and tamponade used. In our work, we found no difference in final BCVA between eyes receiving ICG or BBG stain (Mann-Whitney $U$-test). Previously, a meta-analysis indicated that BBG could contribute to better visual outcome than other dyes for MH surgery but does not significantly influence the closure rate [20]. Second, the work is a retrospective study and included limited cases with relatively short follow-ups. Third, in spite of the favorable anatomic and functional recovery, we could not definitely conclude our technique is better than the classic inverted ILM flap technique, which calls for a randomized controlled study to verify. In conclusion, we present a new technique for large MH called non-inverted pedicle ILM transposition. This technique provides several advantages including relief of macular traction by ILM peeling, remain of physiological ILM, and a promising anatomical and functional recovery. In future, further long-term follow-up is needed to determine the efficacy of this technique. Furthermore, randomized controlled trail is need to explore the physiological role of pedicle ILM in recovering the visual function for patient with large $\mathrm{MH}$. 
Table 2 Functional change pre- and post operation

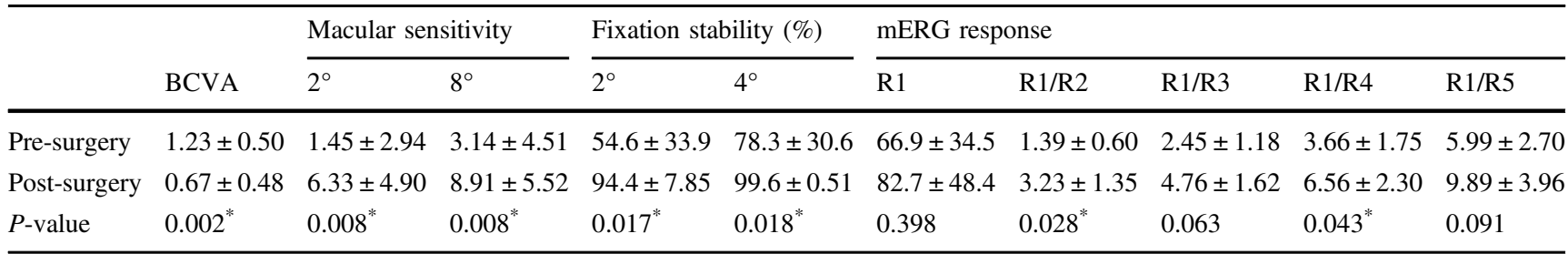

$B C V A$ best-corrected visual acuity, $m E R G$ multifocal electroretinogram, $R$ ring

\section{Summary}

\section{What was known before}

- Surgical closure rate is challenging in cases of large macular holes.

- The inverted ILM flap technique has been reported effective.

\section{What this study adds}

- This new technique first creates a pedicle internal limiting membrane (ILM) flap.

- The pedicle ILM is attached to the superior temporal retina and then transposed to cover the large macular hole in a non-inverted way.

Acknowledgements We thank all those patients with large macular hole, who were willing to undertake the new non-inverted pedicle ILM technique, and those who helped to perform the examination and are not listed as co-authors.

Funding This study was supported by the Jiangsu Provincial Commission of Health and Family Planning (Grant No. H201609), the Jiangsu Natural Science Foundation (BK20171503), the National Key Project of Research and Development Plan (No. 2017YFA0104101). The funders had no role in study design, data collection and analysis, decision to publish, or preparation of the manuscript.

\section{Compliance with ethical standards}

Conflict of interest The authors declare that they have no conflict of interest.

\section{References}

1. Ullrich S, Haritoglou C, Gass C, Schaumberger M, Ulbig MW, Kampik A. Macular hole size as a prognostic factor in macular hole surgery. Br J Ophthalmol. 2002;86:390-3.

2. Alpatov S, Shchuko A, Malyshev V. A new method of treating macular holes. Eur J Ophthalmol. 2007;17:246-52.

3 . Wong R. Novel surgical technique for closure of large fullthickness macular holes. Retina. 2013;33:1977-9.

4. Michalewska Z, Michalewski J, Adelman RA, Nawrocki J. Inverted internal limiting membrane flap technique for large macular holes. Ophthalmology. 2010;117:2018-25.

5. Andrew N, Chan WO, Tan M, Ebneter A, Gilhotra JS. Modification of the inverted internal limiting membrane flap technique for the treatment of chronic and large macular holes. Retina. 2016;36:834-7.

6. Chakrabarti M, Benjamin P, Chakrabarti K, Chakrabarti A. Closing macular holes with "macular plug" without gas tamponade and postoperative posturing. Retina. 2017;37:451-9.

7. Park SW, Pak KY, Park KH, Kim KH, Byon IS, Lee JE. Perfluoro-n-octane assisted free internal limiting membrane flap technique for recurrent macular hole. Retina. 2015;35:2652-6.

8. Hood DC, Bach M, Brigell M, Keating D, Kondo M, Lyons JS, et al. ISCEV guidelines for clinical multifocal electroretinography (2007 edition). Doc Ophthalmol. 2008;116:1-11.

9. Brooks HL. Macular hole surgery with and without internal limiting membrane peeling. Ophthalmology. 2000;107:1939-48.

10. Casini G, Mura M, Figus M, Loiudice P, Peiretti E, De Cilla S, et al. Inverted internal limiting membrane flap technique for macular hole surgery without extra manipulation of the flap. Retina. 2017:37:2138-44.

11. Michalewska Z, Michalewski J, Dulczewska-Cichecka K, Adelman RA, Nawrocki J. Temporal inverted internal limiting membrane flap technique versus classic inverted internal limiting membrane flap technique: a comparative study. Retina. 2015;35:1844-50.

12. Shin MK, Park KH, Park SW, Byon IS, Lee JE. Perfluoro-noctane-assisted single-layered inverted internal limiting membrane flap technique for macular hole surgery. Retina. 2014;34:1905-10.

13. Gelman R, Stevenson W, Prospero Ponce C, Agarwal D, Christoforidis JB. Retinal damage induced by internal limiting membrane removal. J Ophthalmol. 2015;2015:939748.

14. Kenawy N, Wong D, Stappler T, Romano MR, Das RA, Hebbar $\mathrm{G}$, et al. Does the presence of an epiretinal membrane alter the cleavage plane during internal limiting membrane peeling? Ophthalmology. 2010;117:320-3. e321.

15. Steel DHW, Dinah C, White K, Avery PJ. The relationship between a dissociated optic nerve fibre layer appearance after macular hole surgery and Muller cell debris on peeled internal limiting membrane. Acta Ophthalmol. 2017;95:153-7.

16. Ripandelli G, Scarinci F, Piaggi P, Guidi G, Pileri M, Cupo G, et al. Macular pucker: to peel or not to peel the internal limiting membrane? A microperimetric response. Retina. 2015;35:498-507.

17. Tadayoni R, Svorenova I, Erginay A, Gaudric A, Massin P. Decreased retinal sensitivity after internal limiting membrane peeling for macular hole surgery. Br J Ophthalmol. 2012;96:1513-6.

18. Tian T, Chen C, Peng J, Jin H, Zhang L, Zhao P. Novel surgical technique of peeled internal limiting membrane reposition for idiopathic macular holes. Retina. 2017.

19. Lai CC, Chen YP, Wang NK, Chuang LH, Liu L, Chen KJ, et al. Vitrectomy with internal limiting membrane repositioning and autologous blood for macular hole retinal detachment in highly myopic eyes. Ophthalmology. 2015;122:1889-98.

20. Azuma K, Noda Y, Hirasawa K, Ueta T. Brilliant blue g-assisted internal limiting membrane peeling for macular hole: a systematic review of literature and meta-analysis. Retina. 2016;36:851-8. 